\title{
SAR COHERENCE TOMOGRAPHY FOR BOREAL FOREST WITH AID OF LASER MEASUREMENTS
}

\author{
Jaan Praks ${ }^{1}$, Florian Kugler ${ }^{2}$, Juha Hyypp $\ddot{a}^{3}$, Konstantinos Papathanassiou $^{2}$, Martti Hallikainen $^{1}$ \\ ${ }^{1}$ Department of Radio Science and Engineering, Helsinki University of Technology \\ FIN-02015, Espoo, Finland \\ ${ }^{2}$ Microwaves and Radar Institute, German Aerospace Center (DLR) \\ D-82234, Wessling, Germany \\ ${ }^{3}$ Finnish Geodetic Institute \\ FIN-02431, Masala, Finland
}

\begin{abstract}
In this paper we evaluate $\mathrm{X}$ - and L-band SAR coherence tomography in boreal forest with the help of detailed digital terrain and canopy height models, produced by laser scanning. Polarimetric coherence tomography (PCT) needs accurate estimates of ground phase and tree height. Supplemental accurate elevation models allow us to evaluate the performance of PCT in normal case when initial values are derived from RVoG model inversion and provides opportunity to use PCT for nonpolarimetric data. The work is based on E-SAR Lband and X-band measurements in Finland. Our results show that with accurate elevation and tree height information single polarization X-band coherence tomography is feasible and works well. Accurate ground elevation information improves also the performance of fully polarimetric repeat pass L-band PCT. The laser DEM provides better ground phase estimate than RVoG model inversion in the presence of temporal decorrelation. Our results show that accurate ground phase estimation is more critical for successful coherence tomography than other parameters.
\end{abstract}

Index Terms - Polarimetric SAR interferometry, Polarimetric Coherence Tomography, boreal forest, laser, DEM, canopy height.

\section{INTRODUCTION}

Global forest remote sensing can benefit much from SAR instruments. Polarimetric and interferometric SAR techniques can provide terrain model, forest height and even give some information about the inner structure of the canopy. The Random Volume over Ground (RVoG) model [1] forms a basis for many vegetation mapping approaches. Inversion of the RVoG model [2] for fully polarimetric interferometric measurement has been used in several studies to successfully estimate forest height [1], [3] and even X-band single polarization interferometric coherence can be used to invert the RVoG model for forest height [4]. Also a new method [5] to estimate the shape of the scattering function inside the canopy is based partly on the RVoG model. The technique is called Polarization Coherence Tomography (PCT). It approximates the vertical structure function of the canopy with Fourier-Legendre polynomial series. The approximation accuracy is dependent on the available amount of measurements, in this case interferometric baselines. However, calculation of SAR coherency tomography profile requires good estimates for ground phase and tree height as the initial values. Normally the SAR interferometric coherence data is used to calculate both, the vertical profile and required initial values. Unfortunately this approach can be susceptible for propagating errors. In this work we try to evaluate he performance of PCT with the help of supplemental elevation models measured by laser scanner. Additional measurement allow us to evaluate the performance of PCT in normal case when initial values are derived from RVoG model inversion and provides opportunity to use PCT also for single polarization data.

\section{MATERIAL}

The SAR data used in our study was collected during FINSAR campaign [4], carried out in autumn 2003 in Finland. The main instruments of the campaign were E-SAR and HUTSCAT ranging scatterometer. The main campaign took place on 29 September 2003 over the test site in southern Finland (N 60 $11^{\circ}$, E $\left.24^{0} 29^{\prime}\right)$. German E-SAR collected from $3 \mathrm{~km}$ altitude five L-band $(1.3 \mathrm{GHz})$ repeat pass fully polarimetric images $(5 \mathrm{~m}, 10 \mathrm{~m}, 12 \mathrm{~m}$ and $0 \mathrm{~m}$ baselines $)$ and an X-band (9.6 GHz) single-pass single-pol (VV) interferometric image pair. The forest in the area is heterogeneous and consists of small stands, fields and lakes. Most forested areas are located on top of small hills. The dominant tree species are Scotch pine, Norwegian spruce, birch and alder.

The laser scanning over part of FINSAR test site was performed on 12 July 2005 using laser scanner Optech ALTM 
3100 with $100 \mathrm{kHz}$ PRF and $1 \mathrm{~km}$ flight altitude and providing 3-4 pts $/ \mathrm{m}^{2}$ point density on the object. The strip adjustment (matching adjacent slight strip data) was made using TerraMatch. Ground hits were classified using TerraScan [6]. Digital Surface Model (DSM) relevant to treetops was obtained by taking the highest point within a 1-m grid and missing points were interpolated by Delaunay triangulation. The canopy height model (CHM) was then obtained by subtracting the Digital Elevetion Model DEM from the corresponding DSM. The crown DSM was calculated by means of the first pulse echo and the DEM with the last pulse echo. The accuracy of the obtained DEM is better than $20 \mathrm{~cm}$ for forested terrain. The CHM includes a $-70 \mathrm{~cm}$ bias in obtained tree heights and about $0.5 \mathrm{~m}$ std error. Information at individual tree level can be derived from CHM using methods depicted in [7].

\section{METHODS}

The PCT method allows to calculate approximation for vertical scattering function inside the volume when ground phase, volume height and one or more complex coherences is known. In order to derive volume height and ground phase, usually RVoG model inversion is used. In our study we derived these values also from high accuracy digital elevation model and digital canopy height model in order to compare their accuracy and influence to PCT. All the supplemental material was first transferred to E-SAR slant range coordinates. The geocoded CHM measured by laser scanner was also filtered to represent the maximum height $h_{100}$ for $10 \mathrm{~m} \times 10 \mathrm{~m}$ area. In this form the canopy height corresponds better to volume height measured by SAR. The $h_{100}$ was transferred to slant range coordinates by using E-SAR range and azimuth geocoding tables. The missing pixels in slant range $h_{100}$ map were recovered by two dimensional interpolation. The unfiltered DEM was converted to slant range by the same means. In order to get ground phase, we wrapped the laser scanner produced DEM to interferometric SAR phase. The ground phase $\phi_{D E M}$ can be represented in terms of SAR vertical wavenumber $\kappa_{z}$ and terrain elevation $h_{D E M}$ as

$$
\phi_{D E M}=\kappa_{z}\left(h_{D E M}+h_{f}\right)+\phi_{f}
$$

where $h_{f}$ and $\phi_{f}$ are unknown parameters. These two parameters were recovered by fitting the DEM generated ground phase to the SAR measured ground phase on open areas. The open areas were chosen by simple coherence value threshold $(\gamma>0.97)$ and the cost function

$$
E=\sum\left|e^{i \phi_{D E M}}-e^{i \phi_{\gamma}}\right|^{2}
$$

was minimized for $h_{f}$ and $\phi_{f}$ by Nelder-Mead simplex method. For fully polarimetric L-band repeat pass coherences the polarization optimization was carried out and optimal coherences closest to ground and tree top were calculated ac-

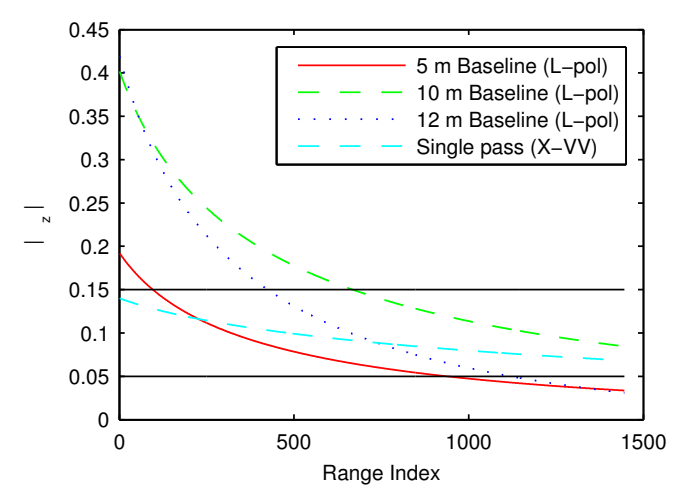

Fig. 1. Vertical wavenumbers $\kappa_{z}$ as a function of range for four baselines available for this study. Black solid lines show the region of feasible $\kappa_{z}$ values for PCT.

cording to RVoG model. The PCT profiles were calculated for slant range coherence images as transects in range direction. The averaging window size for coherence calculation was 10 by 10 pixels in slant range coordinates. For RVoG inversion and PCT we restricted the vertical wavenumber $\kappa_{z}$ value to range where $\pi / 3<h \kappa_{z}<\pi$. For typical forest height we assume that the scattering center is not higher than $20 \mathrm{~m}$ thus we can use threshold values $0.05<\kappa_{z}<0.15$. The wavenumber bigger than these limits can introduce ambiguity to ground phase detection in RVoG inversion and smaller $\kappa_{z}$ areas are too noisy and insensitive to forest height. In Fig 1 is presented $\kappa_{z}$ values along the range of the four available baselines with the feasible value range.

\section{RESULTS AND DISCUSSION}

In Fig 2 is presented a PCT profile for single pass X-band VV-polarization. The ground phase and tree height are obtained from laser scanner DEM and CHM as explained above. The ground phase fitting for X-band single pass data works notably well. The tomogram shows nicely the difference between mixed forest (near range index 760) and the sparse pain forest (near range index 900) where the ground is more visible. In Fig 3 is presented laser measured canopy height map around L-band PCT transect (different area than for X-band). The image is in slant range coordinates. Dark area in the middle of the image is a lake. The yellow line along the $\mathrm{x}$-axis in the middle shows the PCT transect. In Fig 4 is shown the L-band phase of optimized coherences (red stars and blue circles), the phase of the $\mathrm{HH}+\mathrm{VV}$ coherence scattering (magenta dots), ground phase derived from laser DEM (black line), ground phase estimate from RVoG inversion (cyan pluses) and the placement of the treetop (green line). As it is seen, the DEM generated ground phase line fits rather well with the data. The agreement is more clearly visible in open areas nearby. On the lake the phase is random. The scattering 


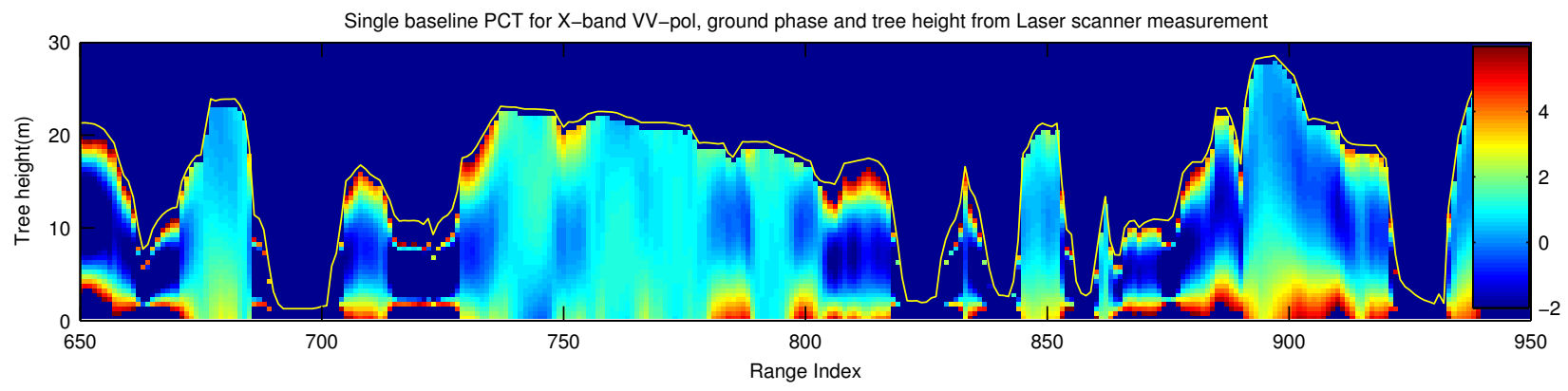

Fig. 2. Coherence tomogram profile for single baseline X-band VV polarization measurement. Ground phase and tree height are obtained from laser scanner measurement. On the $\mathrm{x}$-axis is range index. Note the difference between mixed forest (near range index 760) and the sparse pain forest (near range index 900).

center for optimized coherence is much below the treetops, because the boreal forest is quite sparse. The RVoG model predicted ground phase is noisy, most probably due to temporal decorrelation. For longer baselines where also the temporal baseline is longer, the RVoG model ground phase estimate is even more noisy. In Fig 5 is presented a single baseline PCT for $5 \mathrm{~m}$ baseline fully polarimetric L-band data. In upper two panels calculated with laser measured tree height and ground phase as the initial values, in lower two panels calculated with RVoG model produced tree height and ground phase as the initial values. The PCT with ground phase and tree height values from laser measurement is consistent with forest type. The denser and higher forest between range index 370 and 410 has clearly different profile estimate than lower sparse forest on the left. However in the places where optimized coherence phase values are lower than ground phase, the PCT clearly fails. The RVoG initalized PCT produces more similar profiles for all areas. The temporal decorrelation causes underestimation of the phase and overestimation of the phase difference between lower coherence value and ground phase. This causes RVoG initialized PCT to loose the sensitivity.

\section{CONCLUSIONS}

Our results show that supplemental ground phase and canopy height estimates improve single baseline PCT for repeat pass measurements. Additional ground phase information compensates temporal decorrelation induced ground phase estimate errors. The right initial ground phase estimate is very important for PCT. Errors in initial tree height have smaller effect on resulting PCT profile than poor phase estimate. Additional ground model makes also possible the calculation of coherence tomograms for non-polarimetric single baseline SAR data. The X-band seems to have enough penetration depth in boreal forest to produce good PCT.

\section{REFERENCES}

[1] K.P. Papathanassiou and S.R. Cloude,"Single Baseline Polarimetric SAR Interferometry", IEEE Transactions on Geoscience and Remote Sensing, vol. 39, no. 11, pp. 2352-2363, 2001.

[2] S.R. Cloude and K.P., Papathanassiou, "Three-stage inversion process for polarimetric SAR interferometry", IEE Proceedings - Radar Sonar and Navigation, vol. 150, no. 3, pp. 125-134, 2003.

[3] M. Brandfass, C. Hofmann, J.C. Mura, K.P Papathanassiou, "Polarimetric SAR interferometry as applied to fully polarimetric rain forest data", IEEE Geoscience and Remote Sensing Symposium IGARSS '01, 9-13 July 2001, Proceedings IEEE International, vol. 6, pp. 25752577, 2001.

[4] J. Praks, F. Kugler, K. Papathanassiou, I. Hajnsek, M. Hallikainen "Height estimation of boreal Forest: interferometric model based inversion at L- and X-band vs. HUTSCAT profiling scatterometer" IEEE Transactions on Geoscience and Remote Sensing Letters, no. 3, pp. vol. 4, 466-470, July 2007

[5] S. R. Cloude, "Polarization Coherence Tomography", Radio Science, 41, RS4017 doi:10.1029/2005RS003436, September 2006.

[6] P. Axelsson, "DEM generation from laser scanner data using adaptive TIN models", In International Archives of Photogrammetry and Remote Sensing, 33, Part B4, pp. 110-117, 2000.

[7] J. Hyyppä, Inkinen, M., "Detecting and estimating attributes for single trees using laser scanner", The Photogrammetric Journal of Finland, 16, pp. 27-42, 1999. 


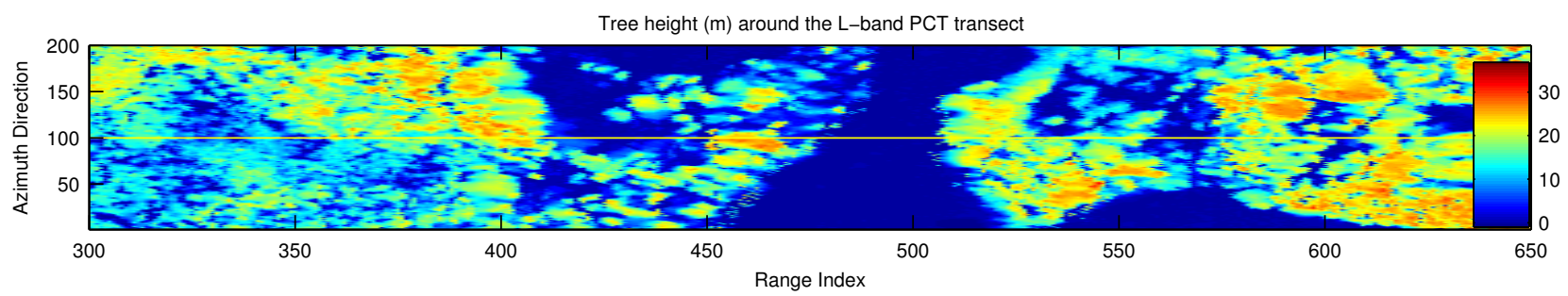

Fig. 3. Tree height map around L-band Polarimetric Coherence Tomography (PCT) track. The track is marked by yellow line.

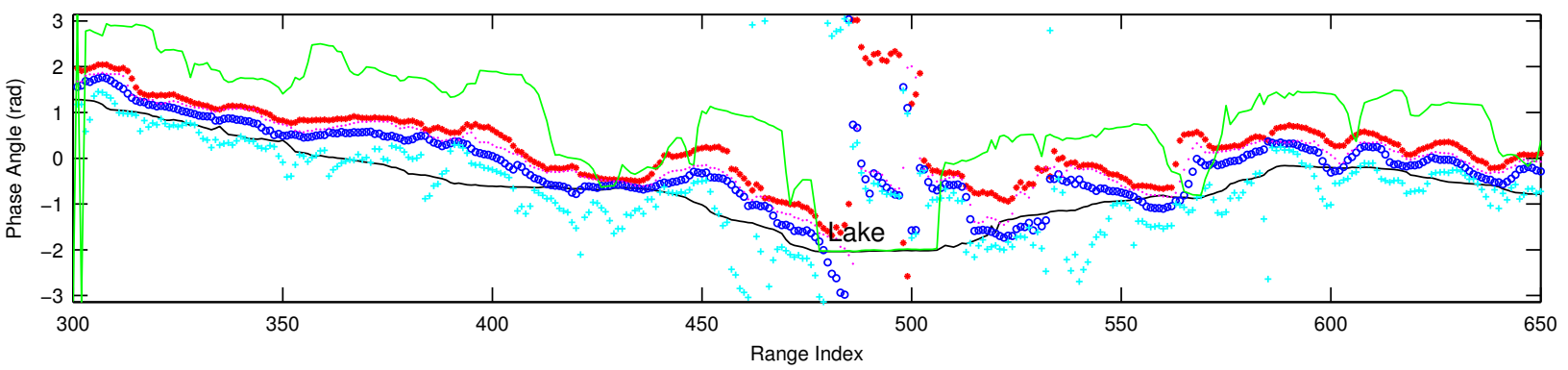

Fig. 4. Estimated ground phase $\phi_{0}$ and coherence phases along the PCT track. The black line represents laser DEM generated ground phase, the red star and the blue circle are optimized coherence phases, the cyan plus stands for RVoG model estimated ground phase, the magenta dot shows HH+VV coherence, the green line marks treetop line in phase scale.
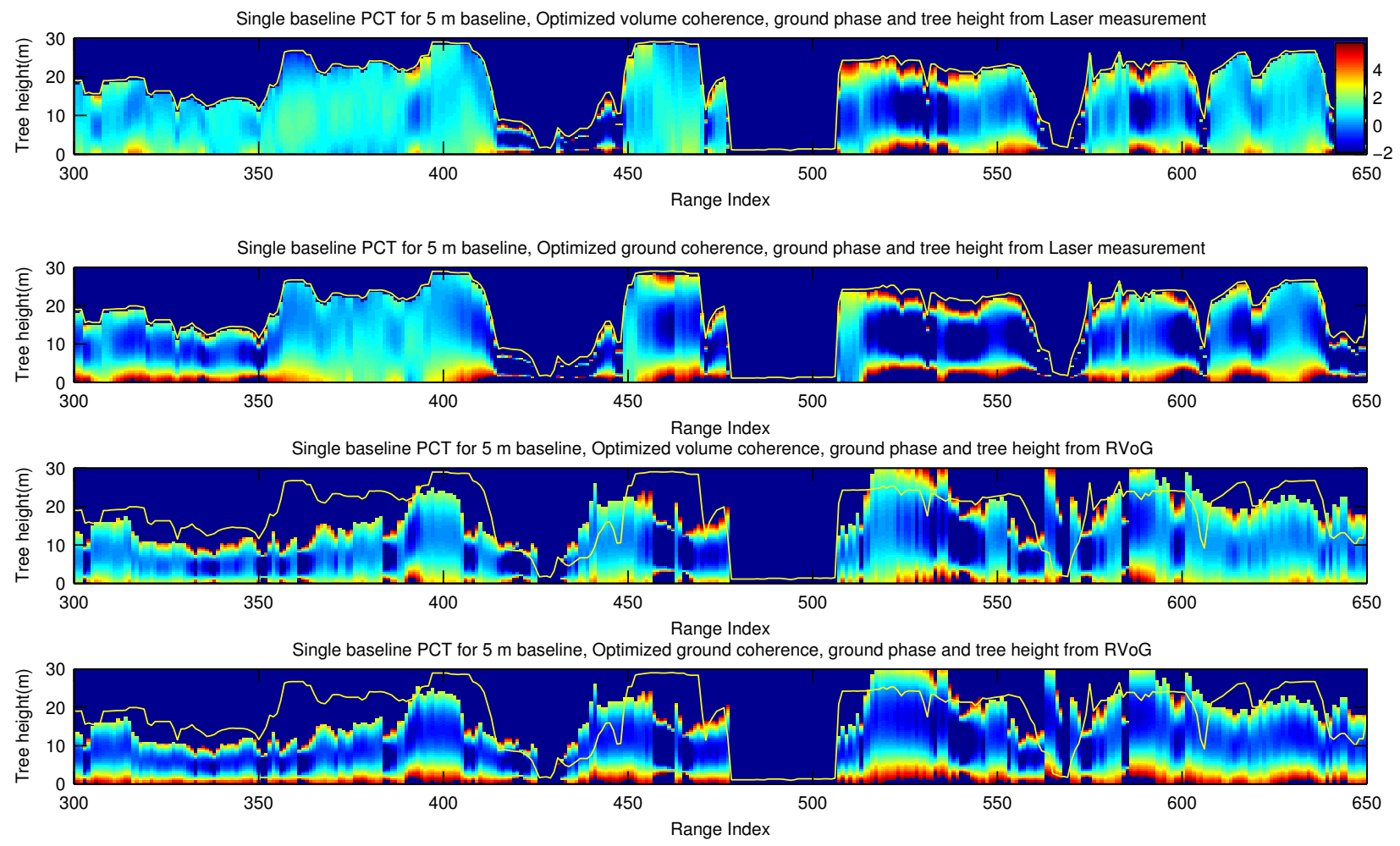

Fig. 5. Single baseline Polarimetric Coherence Tomograms (PCT) for L-band $5 \mathrm{~m}$ baseline optimal coherences with two different initial value sets. PCT in upper two panels are calculated by using laser measured DEM and tree height, lower two panels are calculated by using ground phase and tree height derived by RVoG model inversion. Yellow line represents laser measured tree height $(\mathrm{m})$ in all panels. All the panels have same color scale. 